\title{
MB/BacT Alert
}

National Cancer Institute

\section{Source}

National Cancer Institute. MB/BacT Alert. NCI Thesaurus. Code C88115.

A proprietary, automated microbial growth and detection system optimized for detection of Mycobacterium. 